\title{
Clinical significance of chicken ovalbumin upstream promoter-transcription factor II expression in human colorectal cancer
}

\author{
SUNG-WON SHIN ${ }^{1,2}$, HYUK-CHAN KWON ${ }^{3}$, MEE-SOOK RHO ${ }^{1,4}$, \\ HONG-JO CHOI ${ }^{5}$, JONG-YOUNG KWAK ${ }^{1,2}$ and JOO-IN PARK ${ }^{1,2}$, \\ ${ }^{1}$ Medical Research Center for Cancer Molecular Therapy, Dong-A University; Departments of ${ }^{2}$ Biochemistry, \\ ${ }^{3}$ Internal Medicine, ${ }^{4}$ Pathology and ${ }^{5}$ Surgery, Dong-A University College of Medicine, Busan, Korea
}

Received May 15, 2008; Accepted September 9, 2008

DOI: 10.3892/or_00000195

\begin{abstract}
Chicken ovalbumin upstream promoter-transcription factor II (COUP-TFII) plays an essential role in angiogenesis and development. A previous study showed that the expression of COUP-TFII enhanced invasiveness of human lung carcinoma cells. However, no published data are available concerning the biological and clinical significance of COUP-TFII expression in colorectal cancer. Thus, our objective was to explore the expression of COUPTFII in colorectal cancer as well as its association with clinicopathological features, and to evaluate the role of COUP-TFII as a prognostic indicator in colorectal cancer. We investigated the presence of COUP-TFII in human colorectal cancer tissues and adjacent normal tissues from 95 primary colorectal cancer patients by immunohistochemistry. The correlation between the expression of COUPTFII and clinicopathologic features was investigated. The 3year disease-free survival (DFS) and overall survival (OS) of patients with tumors expressing different levels of COUPTFII were evaluated by the Kaplan-Meier method. No significant correlation was found between COUP-TFII expression and age at surgery, gender, histopathologic differentiation, vessel invasion, carcinoembryonic antigen (CEA), or nodal involvement. However, survival analysis showed that the COUP-TFII-positive group had a significantly better OS compared to COUP-TFII-negative group ( $80.4 \%$ vs. $57.7 \%, \mathrm{P}=0.0491$ ). Based on our results, COUPTFII may represent a biomarker for good prognosis in colorectal cancer.
\end{abstract}

Correspondence to: Dr Joo-In Park, Department of Biochemistry and Medical Research Center for Cancer Molecular Therapy, Dong-A University College of Medicine, 3 Ga 1, DongdaesinDong, Seo-Gu, Busan 602-714, Korea

E-mail: jipark@dau.ac.kr

Key words: colorectal cancer, chicken ovalbumin upstream promoter-transcription factor II, prognosis

\section{Introduction}

Colorectal cancer is the third most-common cancer in the world with one million new cases diagnosed in 2002, and the number of new cases worldwide has increased rapidly since 1975 (1). Tumor invasion, regional lymph node metastasis, and distant organ metastasis are important factors for determining colorectal cancer prognosis. Recent molecular studies have elucidated some of the mechanisms and the sequence of tumor progression $(2,3)$. It is therefore fundamentally important to identify molecular markers of more aggressive colorectal tumor phenotypes in order to adjust patient's treatment accordingly. However, there is a paucity of molecular predictive indicators for regional disease invasion and metastasis.

Chicken ovalbumin upstream promoter-transcription factors (COUP-TFs) are orphan nuclear receptors (4). They belong to the steroid/thyroid hormone receptor superfamily of nuclear receptor proteins and are required for regulation of gene expression (5), development, differentiation, and homeostasis (6). COUP-TFs bind to cis-acting elements in the target genes either as homodimers or as a heterodimer with retinoid X receptors (RXRs) (7). There is evidence that COUP-TFs modulate the activity of other nuclear receptors including estrogen receptors, retinoic acid receptors, RXRs, vitamin D3 receptors, thyroid hormone receptors, peroxisome proliferator activated receptors and hepatocyte nuclear factor 4 , all of which are involved in cell growth and differentiation (7-9). Angiogenesis in COUP-TFII mutant mice is largely impaired $(10,11)$, and defects mimic the phenotypes exhibited by mice lacking angiopoietin-1 or its receptor, TIE2. It has also been suggested that COUP-TFII plays an important role in mesenchymal-endothelial interactions (11). COUP-TFs are expressed in certain tumor cell lines (12), including human endometrial cancer cells (13), lung cancer cells (14), and in adrenal tumors (15), but are not expressed in terminally differentiated epithelial cells. However, the relationship between COUP-TFII expression and cancer development is not fully understood. A recent study has shown that expression of COUP-TFII enhances invasiveness of human lung carcinoma cells and has suggested that COUP-TFII may be considered as a new 
target for anticancer therapies (16). In addition, More et al reported that mitogenic substances which activate the MAP kinase pathway can induce COUP-TFII expression and suggested that an active MAP kinase pathway is essential for COUP-TFII expression in human breast cancer cells (17). In contrast, Lin et al have shown that the expression of COUPTFII plays a role in mediating the growth inhibitory effect of trans-retinoic acid in cancer cells (18). In addition, a previous report demonstrated that the expression of COUP-TFII was reduced in $30 \%$ of breast cancer cell lines and the introduction of COUP-TFII to MDA-MB-435 cells resulted in reduced growth and plating efficiency (19). Although there are some studies on the significance of COUP-TFII expression in several cancer cell lines, the exact role of COUP-TFII on carcinogenesis and tumor cell growth is still controversial and unclear.

The link between COUP-TFII and cancer is suggested by in vitro observation, which shows that the increased expression of COUP-TFII correlated with invasiveness in human lung carcinoma cells (16). However, there is no report on the biological and clinical significance of COUPTFII in colorectal cancer patients. In this study, we investigated the expression of COUP-TFII in 95 primary colorectal cancers and compared this value with that of the corresponding normal colorectal tissue, and looked for correlations between the expression of this protein and clinicopathological factors and prognosis in colorectal cancer patients.

\section{Materials and methods}

Patients and tissue samples. Ninety-five consecutive colorectal cancer patients who were eligible and received surgery at Dong-A University Hospital from 2002 to 2003 were enrolled in the study. Tissue samples from patients were formalin-fixed and paraffin-embedded. Patients with familial adenomatous polyposis or inflammatory bowel disease, synchronous colorectal or extracolorectal cancers, and those lost to follow-up were excluded. None of the patients had a family history of colorectal cancer. Tumor stage was classified according to Dukes' criteria. Fifty-three patients were classified as Dukes' B, and 42 as Dukes' C. Information concerning age, gender, histologic grade, and stage was retrieved by reviewing the pathology and surgical reports. No patient had received preoperative chemotherapy or radiotherapy. This laboratory study was approved by the Institutional Review Board at Dong-A University Hospital, and written informed consent was obtained.

Immunohistochemistry. Immunohistochemical studies for COUP-TFII were performed on formalin-fixed, paraffinembedded, $4 \mu \mathrm{m}$-thick tissue sections using the avidin-biotinperoxidase complex method. The primary antibodies were a mouse monoclonal antibody against COUP-TFII (Perseus Proteomics Inc., Tokyo, Japan) at a 1:1,000 dilution. Deparaffinization of all the sections was performed through a series of xylene baths, and rehydration was performed with a series of graded alcohol solutions. To enhance the immunoreactivity, microwave antigen retrieval was performed at $750 \mathrm{~W}$ for $30 \mathrm{~min}$ in Tris/EDTA buffer ( $\mathrm{pH}$ 9.0) for COUPTFII. After blocking the endogenous peroxidase activity with
Table I. Correlation between patient characteristics and COUP-TFII expression.

\begin{tabular}{|c|c|c|c|}
\hline & \multicolumn{2}{|c|}{ No. of patients (\%) } & \multirow[b]{2}{*}{ P-value } \\
\hline & COUP-TFII (-) & COUP-TFII (+) & \\
\hline \multicolumn{4}{|l|}{ Age (year) } \\
\hline$\leq 60$ & $18(43.9)$ & $23(56.1)$ & 0.837 \\
\hline$>60$ & $22(40.7)$ & $32(59.3)$ & \\
\hline \multicolumn{4}{|l|}{ Gender } \\
\hline Male & $22(43.1)$ & $29(56.9)$ & 0.838 \\
\hline Female & $18(40.9)$ & $26(59.1)$ & \\
\hline \multicolumn{4}{|l|}{ CEA } \\
\hline$\leq 5 \mathrm{ng} / \mathrm{ml}$ & $31(44.3)$ & $39(55.7)$ & 0.638 \\
\hline$>5 \mathrm{ng} / \mathrm{ml}$ & $9(36.0)$ & $16(64.0)$ & \\
\hline \multicolumn{4}{|l|}{ Size } \\
\hline$\leq 5 \mathrm{~cm}$ & $19(38.8)$ & $30(61.2)$ & 0.538 \\
\hline$>5 \mathrm{~cm}$ & $21(45.7)$ & $25(54.3)$ & \\
\hline \multicolumn{4}{|c|}{ Vessel invasion } \\
\hline- & $11(45.8)$ & $13(54.2)$ & 0.811 \\
\hline+ & $29(40.8)$ & $42(59.2)$ & \\
\hline \multicolumn{4}{|l|}{ Site } \\
\hline Colon & $18(40.8)$ & $32(64.0)$ & 0.219 \\
\hline Rectum & $22(48.9)$ & $23(51.1)$ & \\
\hline \multicolumn{4}{|c|}{ Differentiation } \\
\hline Well & $24(40.7)$ & $35(59.3)$ & 0.846 \\
\hline Moderate & $13(46.4)$ & $15(53.6)$ & \\
\hline Poorly & $3(37.5)$ & $5(62.5)$ & \\
\hline \multicolumn{4}{|l|}{$\mathrm{T}$} \\
\hline 1,2 & $4(28.6)$ & $10(71.4)$ & 0.382 \\
\hline 3,4 & $36(44.4)$ & $45(55.6)$ & \\
\hline \multicolumn{4}{|l|}{$\mathrm{N}$} \\
\hline- & $20(37.7)$ & $33(62.3)$ & 0.404 \\
\hline+ & $20(47.6)$ & $22(52.4)$ & \\
\hline
\end{tabular}

CEA, carcinoembryonic antigen and COUP-TFII, chicken ovalbumin upstream promoter-transcription factor II. ${ }^{\mathrm{P}}$ value is based on Fisher's exact test.

$5 \%$ hydrogen peroxidase for $10 \mathrm{~min}$, primary antibody incubation was performed for $1 \mathrm{~h}$ at room temperature. Cap-Plus Biotinylated secondary antibody (Zymed) was applied for $30 \mathrm{~min}$ at room temperature and then Cap-Plus Streptavidin-HRP (Zymed) was applied for $30 \mathrm{~min}$ at room temperature. After washing the tissue samples in Trisbuffered saline for $10 \mathrm{~min}, 3$, 3'-diaminobenzidine was used as a chromogen, and then Mayer's hematoxylin counterstain was applied.

Immunoreactivities for the COUP-TFII expression were defined by the presence of nuclear staining. Samples were defined as COUP-TFII positive if $>5 \%$ of the tumor cells showed immunoreactivity. 
A

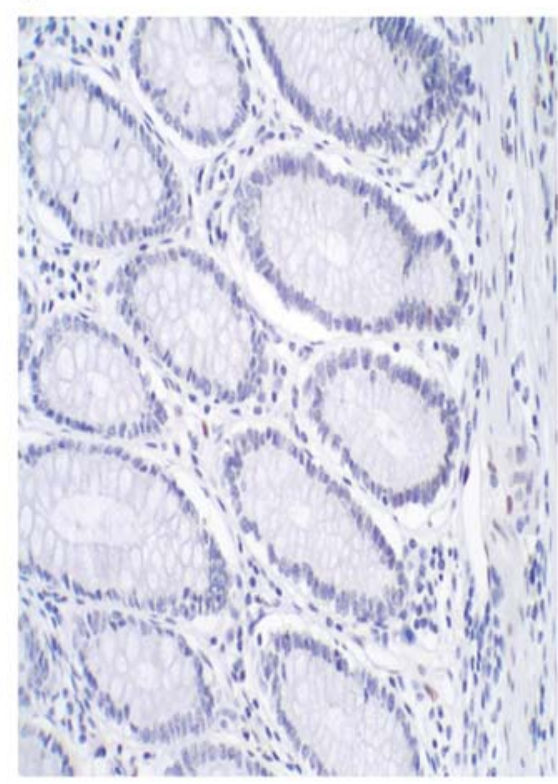

B

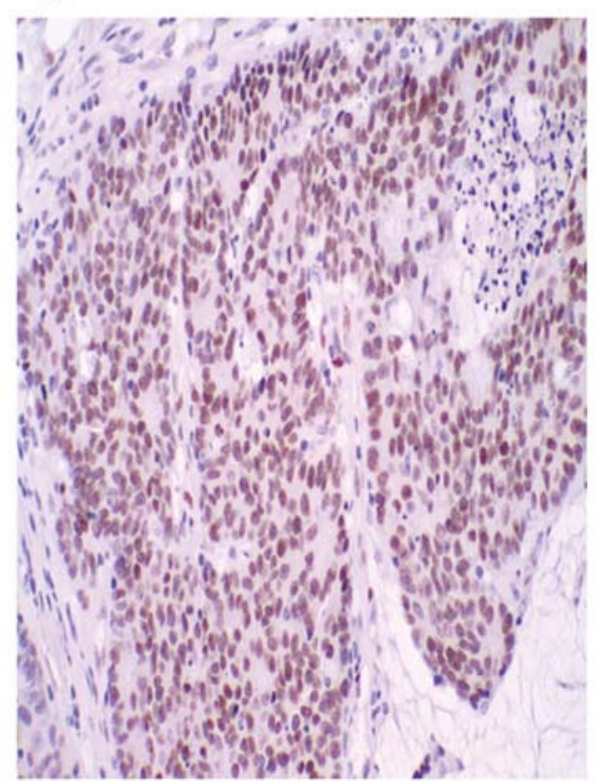

Figure 1. Immunohistochemical staining for COUP-TFII in normal colorectal epithelium and adjacent cancer tissue. A, immunohistochemistry for COUPTFII protein in normal colorectal epithelium (x200). B, immunohistochemistry for COUP-TFII protein in colorectal cancer tissue (x200). In normal colorectal epithelium, COUP-TFII protein was not detected in the nuclei of epithelial cells, however, COUP-TFII protein was strongly stained in the nuclei of colorectal cancer epithelial cells.

A

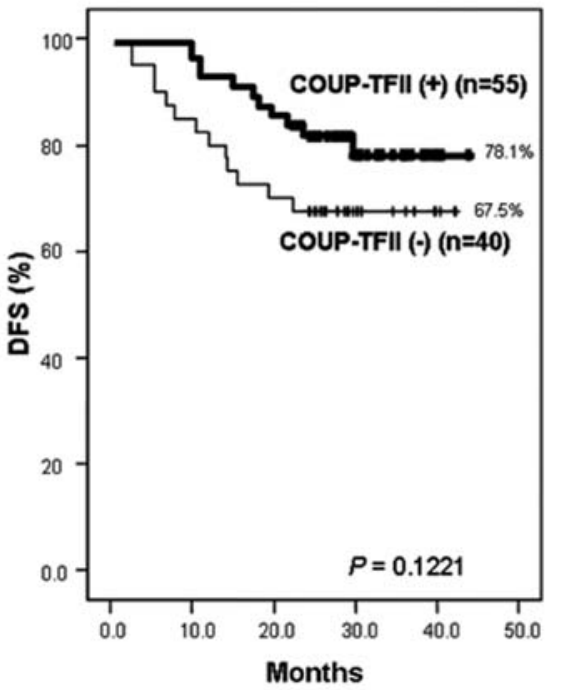

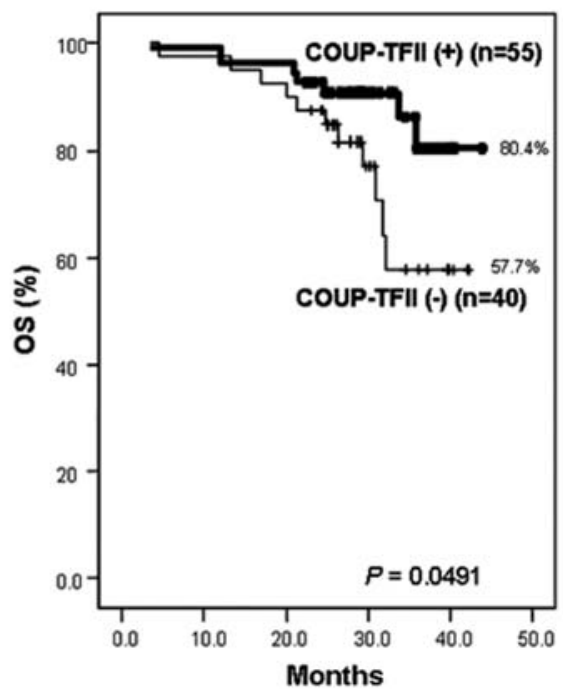

Figure 2. Kaplan-Meier survival curves for 3-year disease-free survival (DFS) and overall survival (OS) in 95 colorectal cancer patients according to expression levels of COUP-TFII. A, patients were dichotomized according to COUP-TFII expression. Three-year disease-free survival (DFS) rates are indicated. B, patients were dichotomized according to COUP-TFII expression. Three-year overall survival (OS) rates are indicated.

Statistical analysis. The samples were divided into two groups on the basis of positive or negative COUP-TFII staining. The number of samples in each group was compared, as were the clinicopathological variables and patient survival rates. Statistical analysis was performed with Fisher's exact test as a $5 \%$ level of significance. Disease-free survival (DFS) was defined as the length of time from surgery to initial disease recurrence. Overall survival (OS) was calculated from the day the surgery was performed to the day of death or the day of the last follow-up. The KaplanMeier method was used for survival analysis, and the statistical significance was analyzed by the log-rank test. Multivariate analysis with covariates showing statistical significance in univariate analysis was performed by the Cox proportional hazards model. A probability level of $\leq 0.05$ was chosen for statistical significance. 
Table II. Three-year DFS and OS according to prognostic factors (univariate analysis).

\begin{tabular}{|c|c|c|c|c|c|}
\hline & No. & $\begin{array}{c}\text { 3-year } \\
\text { DFS (\%) }\end{array}$ & P-value ${ }^{a}$ & $\begin{array}{l}3 \text {-year } \\
\text { OS (\%) }\end{array}$ & P-value \\
\hline \multicolumn{6}{|l|}{ Gender } \\
\hline Male & 51 & 74.3 & 0.9529 & 70.9 & 0.4706 \\
\hline Female & 44 & 62.7 & & 72.0 & \\
\hline \multicolumn{6}{|l|}{ Age (year) } \\
\hline$\leq 60$ & 41 & 78.5 & 0.2724 & 80.8 & 0.2892 \\
\hline$>60$ & 54 & 70.4 & & 64.7 & \\
\hline \multicolumn{6}{|l|}{ Size } \\
\hline$\leq 5 \mathrm{~cm}$ & 49 & 73.2 & 0.8799 & 74.2 & 0.8116 \\
\hline$>5 \mathrm{~cm}$ & 46 & 73.8 & & 70.5 & \\
\hline \multicolumn{6}{|l|}{ CEA } \\
\hline$\leq 5 \mathrm{ng} / \mathrm{ml}$ & 70 & 79.9 & 0.0041 & 74.6 & 0.0081 \\
\hline$>5 \mathrm{ng} / \mathrm{ml}$ & 25 & 56.0 & & 61.0 & \\
\hline \multicolumn{6}{|c|}{ Differentiation } \\
\hline Well & 59 & 77.9 & 0.2882 & 66.8 & 0.9038 \\
\hline Moderate & 28 & 62.6 & & 78.2 & \\
\hline Poorly & 8 & 87.5 & & 87.5 & \\
\hline \multicolumn{6}{|l|}{ Site } \\
\hline Colon & 50 & 75.9 & 0.7709 & 71.9 & 0.7868 \\
\hline Rectum & 45 & 71.6 & & 71.8 & \\
\hline \multicolumn{6}{|c|}{ Vessel invasion } \\
\hline- & 71 & 80.2 & 0.0356 & 76.1 & 0.1262 \\
\hline+ & 24 & 53.6 & & 60.8 & \\
\hline \multicolumn{6}{|l|}{ Tumor } \\
\hline 1,2 & 14 & 92.9 & 0.0957 & 85.7 & 0.1676 \\
\hline 3,4 & 81 & 70.2 & & 69.3 & \\
\hline \multicolumn{6}{|c|}{ Node involve } \\
\hline - & 53 & 84.8 & 0.0076 & 82.4 & 0.0147 \\
\hline+ & 42 & 59.7 & & 57.0 & \\
\hline \multicolumn{6}{|c|}{ COUP-TFII } \\
\hline- & 40 & 67.5 & 0.1221 & 57.7 & 0.0491 \\
\hline+ & 55 & 78.1 & & 80.4 & \\
\hline
\end{tabular}

DFS, disease-free survival; OS, overall survival; CEA, carcinoembryonic antigen; and COUP-TFII, chicken ovalbumin upstream promotertranscription factor II. ${ }^{\mathrm{P}} \mathrm{P}$-value is based on a univariate two-sided log-rank test.

\section{Results}

Expression of COUP-TFII in human colorectal cancer tissues. COUP-TFs are expressed in some tumor cell lines (12), including human endometrial cancer cells (13), lung cancer cells (14), and in adrenal tumors (15), but are not expressed in terminally differentiated epithelial cells. To investigate whether COUP-TFII is expressed in human colorectal carcinomas, immunohistochemistry was performed using an antibody that recognizes an epitope of COUP-TFII.
No or weak COUP-TFII staining was detected in normal colonic mucosa, whereas moderate to strong COUP-TFII staining was observed in the nuclei of colorectal tumor cells (Fig. 1A and B). In the present study, positive expression of COUP-TFII was found in $55(57.89 \%)$ of the 95 colorectal carcinoma tissue specimens, in which the immunostaining occurred predominantly in the nuclei of tumor cells.

Relationship between COUP-TFII expression and clinicopathologic features. The relationship between COUP-TFII 
Table III. Multivariate Cox regression analysis of prognostic factors for outcome in colorectal cancer.

\begin{tabular}{lccc}
\hline Variables & Hazard ratio & $95 \% \mathrm{Cl}$ & P-value \\
\hline Node involvement & 2.449 & $0.901-6.652$ & 0.079 \\
CEA & 3.190 & $1.206-8.434$ & 0.019 \\
COUP-TFII & 0.323 & $0.121-0.862$ & 0.024 \\
\hline
\end{tabular}

CEA, carcinoembryonic antigen and COUP-TFII, chicken ovalbumin upstream promoter-transcription factor II. Multivariate analysis retained only statistically significant $(\mathrm{P}<0.05)$ prognostic factors in the Cox regression model. Variables tested were node involvement, CEA, and COUP-TFII expression level.

expression and clinicopathologic features is summarized in Table I. No significant correlation was found between COUP-TFII expression and age at the time of surgery, gender, tumor location, histopatholgic grade, lymph node metastasis, or vessel invasion.

COUP-TFII expression and outcome in colorectal cancer patients. To determine whether COUP-TFII expression is a significant prognostic factor for the survival of patients with surgically resected colorectal carcinoma, we used a log-rank test with Kaplan-Meier estimates. The median follow-up duration was 29.6 months. Of the 95 patients analyzed, those with COUP-TFII expression (55 cases) had a slightly higher trend of 3-year DFS than those with negative staining (78.1\% vs. $67.5 \%, \mathrm{P}=0.1221$; Table II; Fig. 2A). Patients whose tumors were positive for COUP-TFII expression (55 cases) had a significantly higher 3-year OS than those with negative staining ( $80.4 \%$ vs. $57.7 \%, \mathrm{P}=0.0491$; Table II; Fig. 2B). These results indicate that the elevated COUP-TFII expression is associated with a better tumor prognosis and suggest that COUP-TFII expression may be a valuable good prognostic marker in colorectal cancer.

Multivariate analysis was used to determine the independent prognostic significance of COUP-TFII expression. Nodal involvement and CEA were tested in a Cox proportional hazards model. CEA and COUP-TFII expression were independent prognostic factors $(\mathrm{P}=0.019$ and $\mathrm{P}=0.024$, respectively; Table III). However, nodal involvement was not an independent prognostic factor for colorectal cancer $(\mathrm{P}=0.079$; Table III). The role of COUPTFII in colorectal cancer may be better defined after a longer follow-up period and in a larger sample size. Future prospective studies to evaluate the clinical significance of COUP-TFII expression in colorectal cancer patients are warranted.

\section{Discussion}

Although the expression profile of COUP-TFII in several cancer cell lines has been investigated previously (12-15), no published data are available on the expression of COUPTFII and the role of COUP-TFII in colorectal cancer.
Analyzing 95 colorectal cancer patients by immunohistochemistry, we have shown that $57.89 \%$ of the colorectal cancer cases in our samples were positive for COUP-TFII expression. We did not detect strong expression of COUPTFII in the nuclei of normal colorectal mucosa cells (Fig. 1A). Although studies investigating the expression of COUP-TFII in several cancer cell lines have been reported (12-15), the mechanism underlying different expression levels of COUPTFII in colorectal cancer is currently unknown. Further studies to evaluate the effect of the different levels of COUP-TFII expression in colorectal cancer cell lines are necessary to clarify the target genes and downstream pathways of COUP-TFII activity. In this study, no significant correlation was found between COUP-TFII expression and age at surgery, gender, CEA, size, vessel invasion, tumor location, histopathologic differentiation, or lymph node metastasis (Table I). We compared the 3-year OS rate between the COUP-TFII negative and COUP-TFII positive expression groups. Patients with COUP-TFII positive tumors (55 cases) had a significantly better 3 -year OS rate than those with COUP-TFII negative tumors $(80.4 \%$ vs. $57.7 \%, \mathrm{P}=0.0491$; Table II; Fig. 2B). In addition, multivariate analysis showed that COUP-TFII expression and CEA were independent prognostic factors for colorectal cancer (Table III). However, a longer follow-up may be necessary to clarify this point. The reason why the colorectal cancers with positive COUP-TFII expression show better OS is not known. The physiological role of COUP-TFII is currently elucidated. Gene knockout experiments have revealed that COUP-TFII mutants display defects in angiogenesis, vascular remodeling, and fetal heart development $(10,11)$. A previous study showed that the expression of COUP-TFII enhanced invasive ability and expression of collagenase type IV (MMP-2) of human lung carcinoma cells. Based on those observations, we expected that the patients with COUP-TFII-positive expression had poorer prognosis than those with COUP-TFII negative expression. However, our data are contradictory to our expectation. The expression of COUP-TFII was not related to vessel invasion or nodal involvement. The reason for the discrepancies between our study and the previous data may result from the different tissues and different cells studied. The findings support our data are as follows. Lin et al have shown that the expression of COUP-TFII plays a role in mediating the growth inhibitory effect of trans-retinoic acid in cancer cells (18). A previous report demonstrated that the expression of COUP-TFII was reduced in $30 \%$ of breast cancer cell lines and the introduction of COUP-TFII to MDA-MB-435 cells resulted in reduced growth and plating efficiency (19). To investigate the biological function of COUP-TFII, we are planning the following experiments: a) Establishment of the transfectant of the COUP-TFII gene to clarify the effect of proliferation and invasiveness of human colorectal cancer cells. b) Interference of the COUP-TFII gene expression by small interfering RNA to examine the alteration of its proliferation and invasiveness.

Currently, the biologic function and significance of COUP-TFII in various cancers remains unknown. We are investigating the expression and biological significance of COUP-TFII in several cancer tissues, including esophageal 
and gastric cancers. In summary, we have demonstrated COUP-TFII protein expression in colorectal cancers using immunohistochemistry. Positive COUP-TFII protein expression was detected in $57.89 \%$ of colorectal cancers in our sample; however, COUP-TFII protein expression did not correlate with any clinicopathological parameters evaluated. The study demonstrated that COUP-TFII is a potential positive prognostic marker. To our knowledge, this is the first study to determine that COUP-TFII protein expression level in primary colorectal cancers is predictive of good prognosis. The molecular classification of COUP-TFII expression in primary colorectal cancers may be useful as an indicator for good prognosis. Additional studies on biological behavior of the colorectal cancers expressing COUP-TFII may allow the development of new targeted therapies to enhance COUPTFII activity.

\section{Acknowledgements}

This work was supported by the Korea Science and Engineering Foundation through the Medical Research Center for Cancer Molecular Therapy at Dong-A University (R13-2002-044-05002-0).

\section{References}

1. Parkin DM, Bray F, Ferlay J, et al: Global cancer statistics, 2002. CA Cancer J Clin 55: 74-108, 2005.

2. Goel A, Arnold CN and Boland CR: Multistep progression of colorectal cancer in the setting of microsatellite instability: new details and novel insights. Gastroenterology 121: 1497-1502, 2001.

3. Zhou W, Goodman SN, Galizia G, et al: Counting alleles to predict recurrence of early-stage colorectal cancers. Lancet 359: 219-225, 2002

4. Smirnov DA, Hou S, Liu X, et al: COUP-TFII is up-regulated in adenovirus type 12 tumorigenic cells and is a repressor of MHC class I transcription. Virology 284: 13-19, 2001.

5. Navab R, Wang Y, Chow YH, et al: Regulation of human Clara cell $10 \mathrm{kD}$ protein expression by chicken ovalbumin upstream promoter transcription factors (COUP-TFs). Am J Respir Cell Mol Biol 27: 273-285, 2002.

6. Tsai MJ and O'Malley BW: Molecular mechanisms of action of steroid/thyroid receptor superfamily members. Annu Rev Biochem 63: 451-486, 1994.
7. Mangelsdorf DJ and Evans RM: The RXR heterodimers and orphan receptors. Cell 83: 841-850, 1995.

8. Klinge CM, Silver BF, Driscoll MD, et al: Chicken ovalbumin upstream promoter-transcription factor interacts with estrogen receptor, binds to estrogen response elements and half-sites, and inhibits estrogen-induced gene expression. J Biol Chem 272: 31465-31474, 1997.

9. Nakshatri H and Bhat-Nakshatri P: Multiple parameters determine the specificity of transcriptional response by nuclear receptors HNF-4, ARP-1, PPAR, RAR and RXR through common response elements. Nucleic Acids Res 26: 2491-2499, 1998.

10. Pereira FA, Qiu Y, Zhou G, et al: The orphan nuclear receptor COUP-TFII is required for angiogenesis and heart development. Genes Dev 13: 1037-1049, 1999.

11. Zhou C, Tsai SY and Tsai MJ: From apoptosis to angiogenesis: new insights into the roles of nuclear orphan receptors, chicken ovalbumin upstream promoter-transcription factors, during development. Biochim Biophys Acta 1470: M63-M68, 2000.

12. Kieback DG, Runnebaum IB, Moebus VJ, et al: Chicken ovalbumin upstream promoter-transcription factor (COUP-TF): an orphan steroid receptor with a specific pattern of differential expression in human ovarian cancer cell lines. Gynecol Oncol 51: $167-170,1993$

13. Kieback DG, Levi T, Kohlberger P, et al: Chicken ovalbumin upstream promoter-transcription factor (COUP-TF) expression in human endometrial cancer cell lines. Anticancer Res 16: 3371-3376, 1996

14. Wu Q, Li Y, Liu R, et al: Modulation of retinoic acid sensitivity in lung cancer cells through dynamic balance of orphan receptors nur77 and COUP-TF and their heterodimerization. EMBO J 16: 1656-1669, 1997

15. Shibata H, Ando T, Suzuki T, et al: Differential expression of an orphan receptor COUP-TFI and corepressors in adrenal tumors. Endocr Res 24: 881-885, 1998.

16. Navab R, Gonzalez-Santos JM, Johnston MR, et al: Expression of chicken ovalbumin upstream promoter-transcription factor II enhances invasiveness of human lung carcinoma cells. Cancer Res 64: 5097-5105, 2004.

17. More E, Fellner T, Doppelmayr H, et al: Activation of the MAP kinase pathway induces chicken ovalbumin upstream promotertranscription factor II (COUP-TFII) expression in human breast cancer cell lines. J Endocrinol 176: 83-94, 2003.

18. Lin F, Kolluri SK, Chen G, et al: Regulation of retinoic acidinduced inhibition of AP-1 activity by orphan receptor chicken ovalbumin upstream promoter-transcription factor. J Biol Chem 277: 21414-21422, 2002.

19. Nakshatri H, Mendonca MS, Bhat-Nakshatri P, et al: The orphan receptor COUP-TFII regulates $\mathrm{G} 2 / \mathrm{M}$ progression of breast cancer cells by modulating the expression/activity of p21WAF1/CIP1, cyclin D1, and cdk2. Biochem Biophys Res Commun 270: 1144-1153, 2000. 